LA PLATA - TH 96/03

\title{
Approximate flavor symmetries at a grand unification scale
}

\author{
D. Gómez Dumm* \\ Laboratorio de Física Teórica, \\ Departamento de Física, U. N. L. P. \\ c. c. 67,1900 La Plata, Argentina.
}

\begin{abstract}
We study the evolution of fermion mass matrices considering the hypothesis of approximate flavor symmetries (AFS) in the Standard Model and a two-Higgsdoublet model. We find that the hierarchical structure is not significantly altered by the running, hence the assumption of AFS is entirely compatible with a grand unification scenario.
\end{abstract}

Typeset using REVTEX

*Fellow of CONICET (Argentina) 
The presence of strong hierarchies among fermion masses and mixing angles is in general regarded as a signal of new physics beyond the Standard Model (SM). At present, many different models have been developed, searching for a mechanism which could give rise to the observed parameter hierarchies in a "natural" way.

In this work, we will concentrate on a model based on the existence of approximate flavor symmetries (AFS), which has been recently proposed by Antaramian, Hall and Rašin [1]. This model assumes the presence of a set of global $U(1)$ symmetries (one for each quark and lepton flavor) which are only slightly broken, conducing to different suppression factors in the fermion mass matrices. The possible extension to many Higgs doublets [2], as well as the presence of neutrino oscillations [3], have also been studied within this scheme.

Our purpose is to test the possibility of inserting the idea of AFS into the context of a grand unified theory (GUT), where the $S U(3)_{C} \otimes S U(2)_{L} \otimes U(1)_{Y}$ gauge structure of the SM is nothing but the low-energy manifestation of a theory containing a single gauge coupling constant. In such a framework, it is natural to expect that the fermion mass matrix textures will be determined at the scale of grand unification (typically of order $\sim 10^{15} \mathrm{GeV}$ ), rather than the electroweak one. That is what would happen if the gauge and the $U(1)$ flavor symmetries were spontaneously broken at the same time. The form of the mass matrices at the electroweak scale will then be obtained by considering the evolution of the Yukawa couplings through the renormalization group equations (RGE).

Evolution of AFS with the energy scale. In the Standard Model, the Yukawa couplings can be written as

$$
\mathcal{L}_{Y}=\sum_{i, j=1}^{3}\left(\lambda_{i j}^{U} \bar{Q}_{i} U_{j} \frac{\tilde{H}}{\sqrt{2}}+\lambda_{i j}^{D} \bar{Q}_{i} D_{j} \frac{H}{\sqrt{2}}+\lambda_{i j}^{E} \bar{L}_{i} E_{j} \frac{H}{\sqrt{2}}\right)+\text { h.c. }
$$

where $Q_{i}$ and $L_{i}$ are quark and lepton $S U(2)_{L}$ doublets and $U_{j}, D_{j}$ and $E_{j}$ stand for right fermion fields (we assume by now that only one Higgs doublet $H$ is present). The hypothesis of AFS determines the order of magnitude for the coupling parameters $\lambda$, leading to the 
relations

$$
\left|\lambda_{i j}^{U}\right| \approx \epsilon_{Q_{i}} \epsilon_{U_{j}}, \quad\left|\lambda_{i j}^{D}\right| \approx \epsilon_{Q_{i}} \epsilon_{D_{j}}, \quad\left|\lambda_{i j}^{E}\right| \approx \epsilon_{L_{i}} \epsilon_{E_{j}}
$$

where the $\epsilon_{F_{i}}$ 's are dimensionless suppression factors originated with the flavor symmetry breakdown. Their values can be approximately obtained from the experimental data on quark masses and mixing angles.

In refs. [1 3], the relations (2) are assumed to hold at the scale $Q^{2} \sim M_{Z}^{2}$. However, as stated above, the $\lambda$ matrices will evolve with energy. We are interested here in the form of the Yukawa couplings at the large energy scale $M_{X} \sim 10^{15} \mathrm{GeV}$, hence we will consider the one-loop RGE for the $\lambda$ 's in the SM. These can be written as [4]

$$
\frac{d \lambda^{F}}{d t}=-\frac{1}{16 \pi^{2}}\left[G_{F}(t) \mathbb{1}-T_{F}(t) \mathbb{1}-\frac{3}{2} S_{F}(t)\right] \lambda^{F}
$$

where $\mathbb{1}$ and $S_{F}$ (as well as $\lambda^{F}$ ) are matrices in flavor space, with $F=U, D, E$. We have used here the definitions

$$
\begin{aligned}
& t=\frac{1}{2} \ln \left(Q^{2} / M_{Z}^{2}\right) \\
& T_{F}=\operatorname{Tr}\left(3 \lambda^{U} \lambda^{U \dagger}+3 \lambda^{D} \lambda^{D \dagger}+\lambda^{E} \lambda^{E \dagger}\right) \\
& S_{U}=-S_{D}=\lambda^{U} \lambda^{U \dagger}-\lambda^{D} \lambda^{D \dagger}, \quad S_{E}=\lambda^{E} \lambda^{E \dagger} \\
& G_{U}=8 g_{3}^{2}+\frac{9}{4} g_{2}^{2}+\frac{17}{12} g_{1}^{2}, \quad G_{D}=8 g_{3}^{2}+\frac{9}{4} g_{2}^{2}+\frac{5}{12} g_{1}^{2}, \quad G_{E}=\frac{9}{4} g_{2}^{2}+\frac{15}{4} g_{1}^{2}
\end{aligned}
$$

being $g_{1}, g_{2}$ and $g_{3}$ the running coupling constants corresponding to the $U(1)_{Y}, S U(2)_{L}$ and $S U(3)_{C}$ symmetry groups respectively. As it is well known, their evolution is given by

$$
g_{i}^{2}\left(t_{2}\right)=g_{i}^{2}\left(t_{1}\right)\left(1-\frac{K_{i}}{8 \pi^{2}}\left(t_{2}-t_{1}\right) g_{1}^{2}\left(t_{1}\right)\right)^{-1}
$$

with

$$
K_{1}=\frac{20}{9} N+\frac{1}{6}, \quad K_{2}=\frac{4}{3} N-\frac{43}{6}, \quad K_{3}=\frac{4}{3} N-11
$$

( $N=$ number of generations). 
Let us write now the $\lambda$ matrices using their approximate form (2), and ignoring for simplicity the presence of complex phases. From (3), the $\epsilon$ parameters corresponding to $\lambda^{U}$ will evolve with the energy scale according to

$$
-16 \pi^{2} \frac{d}{d t} \ln \left(\epsilon_{Q_{i}} \epsilon_{U_{j}}\right)=\alpha_{U}(t)
$$

with

$$
\alpha_{U}(t) \equiv G_{U}(t)-T_{F}(t)-\frac{3}{2} \sum_{k} \epsilon_{Q_{k}}^{2} \sum_{l}\left(\epsilon_{U_{l}}^{2}-\epsilon_{D_{l}}^{2}\right)
$$

Similar expressions can be easily found for $F=D$ and $E$. In this way, at the scale $t_{X}=$ $1 / 2 \ln \left(M_{X}^{2} / M_{Z}^{2}\right)$ we find

$$
\left(\epsilon_{Q_{i}} \epsilon_{U_{j}}\right)\left(t_{X}\right)=\left(\epsilon_{Q_{i}} \epsilon_{U_{j}}\right)(0) \exp \left(-\frac{1}{16 \pi^{2}} \int_{0}^{t_{X}} \alpha_{U}(t) d t\right)
$$

This means that the running of $\lambda^{U}$ (and equivalently $\lambda^{D, E}$ ) has not changed the ratios between the matrix elements: the sole effect has been the introduction of a global multiplicative constant. On the other hand, if AFS are assumed to hold at the large scale $t_{X}$ (i.e., just after the spontaneous symmetry breakdown), they will be preserved when the coupling constants run down to the electroweak energies. Thus the AFS hypothesis, as presented in ref. [1], should in principle be compatible with a grand unification scheme.

It is worth to notice that the equation (7) is just an approximate result, since we have taken the relations (2) as if they were exact. In fact, this would conduce to rank 1 quark and lepton mass matrices, that is, to two zero mass eigenvalues for each fermion type. The corresponding (order unity) corrections, however, will appear only in the last term of $\alpha_{U}$, being suppressed by the small $\epsilon^{2}$ parameters.

Numerical analysis. Once the mass matrix elements are determined at the scale $Q^{2}=M_{Z}^{2}$, the multiplicative constants $\beta_{F} \equiv \exp \left(-1 /\left(16 \pi^{2}\right) \int_{0}^{t_{X}} \alpha_{F} d t\right)$ can be estimated numerically. For definiteness, we will use the $\epsilon_{Q_{i}} / \epsilon_{Q_{j}}$ ratios given in ref. [2] (we assume that 
they do not vary significantly when running from 1 to $90 \mathrm{GeV}$ ), together with the quark mass values [5] at the scale of the $Z$ mass [ [4]:

$$
\begin{gathered}
\epsilon_{Q_{1}} / \epsilon_{Q_{2}} \approx 0.2 \quad \epsilon_{Q_{2}} / \epsilon_{Q_{3}} \approx 0.04 \\
m_{d} \simeq 5.6 \mathrm{MeV}, \quad m_{s} \simeq 0.11 \mathrm{GeV}, \quad m_{b} \simeq 3.4 \mathrm{GeV} \\
m_{u} \simeq 3.2 \mathrm{MeV}, \quad m_{c} \simeq 0.85 \mathrm{GeV}
\end{gathered}
$$

We will concentrate in the quark sector (the lepton matrix $\lambda^{E}$ approximately decouples in ([])), ignoring as before the presence of complex phases. The matrix elements $\lambda_{i j}^{U, D}$ can be approximately calculated from the values in (10) taking into account the relations [2]

$$
\epsilon_{Q_{i}} \epsilon_{F_{i}} \approx \frac{m_{F_{i}}}{\langle\phi\rangle}
$$

where $\langle\phi\rangle \simeq 175 \mathrm{GeV}$ is the vacuum expectation value (VEV) of the neutral Higgs field.

Our numerical results are represented in figure 1, where the solid lines stand for the values of $\beta_{U}$ and $\beta_{D}$ as functions of the top quark mass. We also include a dashed line, which corresponds to the value of the matrix element $\lambda_{33}^{U}$ at the scale $M_{X}$. This parameter deserves special interest, since it is the largest of the $\lambda_{i j}^{F}$ appearing in the Yukawa couplings. At the electroweak scale, the relation $\lambda_{33}^{U} \simeq m_{t} /\langle\phi\rangle$ conduces to $\lambda_{33}^{U} \approx 1$ for the expected range of $m_{t}$, hence the identification of $\epsilon_{Q_{3}}$ and $\epsilon_{U_{3}}$ as suppression factors is questionable. However, as it is shown in the figure, the situation is considerably improved at the $M_{X}$ scale, where the value of $\lambda_{33}^{U}$ lies between $1 / 3$ and $1 / 2$ for $m_{t}$ varying from 150 to $200 \mathrm{GeV}$. To this respect, notice that although the symbol " $\approx$ " in (11) indicates that the equalities are in general just approximate (within a factor 2 or 3 ), the large value of $m_{t}$ compared to $m_{u, c}$ implies that the relation has to be almost exact for $F=U, i=3$.

Two-Higgs-doublet case. Let us finally carry out a similar analysis for a two-Higgs-doublet model (THDM) with natural flavor conservation (NFC) [6]. In this scheme, the form of the 
Yukawa couplings will be entirely similar to that of eq. (11), except for the replacements $H \rightarrow H_{1}$ and $\tilde{H} \rightarrow \tilde{H}_{2}$. The NFC condition ensures that only one Higgs couples to all the quarks of a given electric charge, preventing in this way the presence of flavor-changing processes at the tree levelf. Notice that one has to deal with two non-zero Higgs VEVs, $v_{1}$ and $v_{2}$, therefore it is usual to introduce a new parameter,

$$
\tan \beta \equiv \frac{v_{2}}{v_{1}}
$$

As before, we will assume the AFS structure (2) and study the evolution of the coupling "constants" with energy. The relevant one-loop RGE for the THDM can be written as in (3) with the definitions [7]

$$
\begin{aligned}
& S_{U}=\lambda^{U} \lambda^{U \dagger}+\frac{1}{3} \lambda^{D} \lambda^{D \dagger}, \quad S_{D}=\frac{1}{3} \lambda^{U} \lambda^{U \dagger}+\lambda^{D} \lambda^{D \dagger} \\
& T_{U}=\operatorname{Tr}\left(3 \lambda^{U} \lambda^{U \dagger}\right), \quad T_{D}=T_{E}=\operatorname{Tr}\left(3 \lambda^{D} \lambda^{D \dagger}+\lambda^{E} \lambda^{E \dagger}\right)
\end{aligned}
$$

being $t, S_{E}$ and $G_{F}$ the same as in (四). The evolution of the $g_{i}$ 's has also the form (5), where the constants $K_{i}$ are now given by

$$
K_{1}=\frac{20}{9} N+\frac{1}{3}, \quad K_{2}=\frac{4}{3} N-7, \quad K_{3}=\frac{4}{3} N-11
$$

In analogy with the Standard Model, it is immediate to find that the evolution of the $\lambda$ matrices does not change the relations between the matrix elements. We have for the THDM

$$
\lambda_{i j}^{F}\left(t_{X}\right)=\lambda_{i j}^{F}(0) \beta_{F}^{(2 h)}=\lambda_{i j}^{F}(0) \exp \left(-\frac{1}{16 \pi^{2}} \int_{0}^{t_{X}} \alpha_{F}^{(2 h)}(t) d t\right)
$$

where

$$
\alpha_{U, D}^{(2 h)}(t) \equiv G_{U, D}(t)-T_{U, D}(t)-\frac{1}{2} \sum_{k} \epsilon_{Q_{k}}^{2} \sum_{l} \epsilon_{(U, D)_{l}}^{2}
$$

\footnotetext{
${ }^{1}$ It has been argued that with the introduction of AFS, the NFC requirement could be avoided. However, in that case further assumptions would be necessary to suppress the appearing CPviolating phases [2].
} 
Consequently, it is plausible to assume that the AFS are originated at a grand unification scale, just like in the SM case.

In order to get numerical estimations for the $\beta_{U, D}^{(2 h)}$ factors, we will take once again the values in (10). However, due to the two-doublet structure of the model, the relations (11) will now be replaced by

$$
\epsilon_{Q_{i}} \epsilon_{D_{i}} \approx \frac{m_{D_{i}}}{v_{1}} \quad \epsilon_{Q_{i}} \epsilon_{U_{i}} \approx \frac{m_{U_{i}}}{v_{2}}
$$

while the VEVs have to satisfy the constraint

$$
\left(v_{1}^{2}+v_{2}^{2}\right)^{\frac{1}{2}} \simeq 175 \mathrm{GeV}
$$

resulting from the electroweak symmetry breakdown. The value of $\tan \beta$ is a new unknown parameter, which could in principle be determined experimentally.

In figure 2, we present our numerical results for $\beta_{U}^{(2 h)}$ (a similar behaviour is found for $\left.\beta_{D}^{(2 h)}\right)$ and $\lambda_{33}^{U}\left(M_{X}\right)$ as functions of $m_{t}$, for different values of $\tan \beta$. It can be seen that if $\tan \beta \lesssim 1$, the value of $\lambda_{33}^{U}$ is less than one only for $m_{t} \sim 150 \mathrm{GeV}$, growing severely as long as $m_{t}$ approaches $170 \mathrm{GeV}$. The regard of $\lambda_{33}^{U}$ as a parameter reflecting an approximate flavor symmetry could be plausible only for large values of $\tan \beta$, where the Yukawa couplings for the up-type quarks are similar to those of the SM. Hence, taking into account the existing phenomenological bounds on $\tan \beta$ [8], the possibility of assuming AFS at the $M_{X}$ scale within the THDM appears to be considerably reduced in comparison with the Standard Model case.

I would like to acknowledge Prof. L. Epele for a critical revision of the manuscript. This work has been partially supported by Universidad Nacional de La Plata (Argentina). 


\section{REFERENCES}

[1] A. Antaramian, L. J. Hall and A. Rašin, Phys. Rev. Lett. 69 (1992) 1871. See also A. Rašin, Approximate flavor symmetries, preprint LBL-35530; UCB-PTH-94/09, April 1994.

[2] L. J. Hall and S. Weinberg, Phys. Rev. D 48 (1993) R979.

[3] A. Rašin and J. P. Silva, Phys. Rev. D 49 (1994) R20.

[4] T. P. Cheng, E. Eichten and L.-F. Li, Phys. Rev. D 9 (1974) 225.

B. Grzadkowski and M. Lindner, Phys. Lett. B193 (1987) 71.

[5] J. Gasser and H. Leutwyler, Phys. Rep. 87 (1982) 27.

[6] S. L. Glashow and S. Weinberg, Phys. Rev. D 15 (1977) 1958.

[7] B. Grzadkowski, M. Lindner and S. Theisen, Phys. Lett. B198 (1987) 64.

[8] See J. F. Gunion, H. E. Haber, G. L. Kane and S. Dawson, The Higgs Hunter Guide (Addison-Wesley, Reading, MA, 1990), and references therein. 


\section{FIGURES}

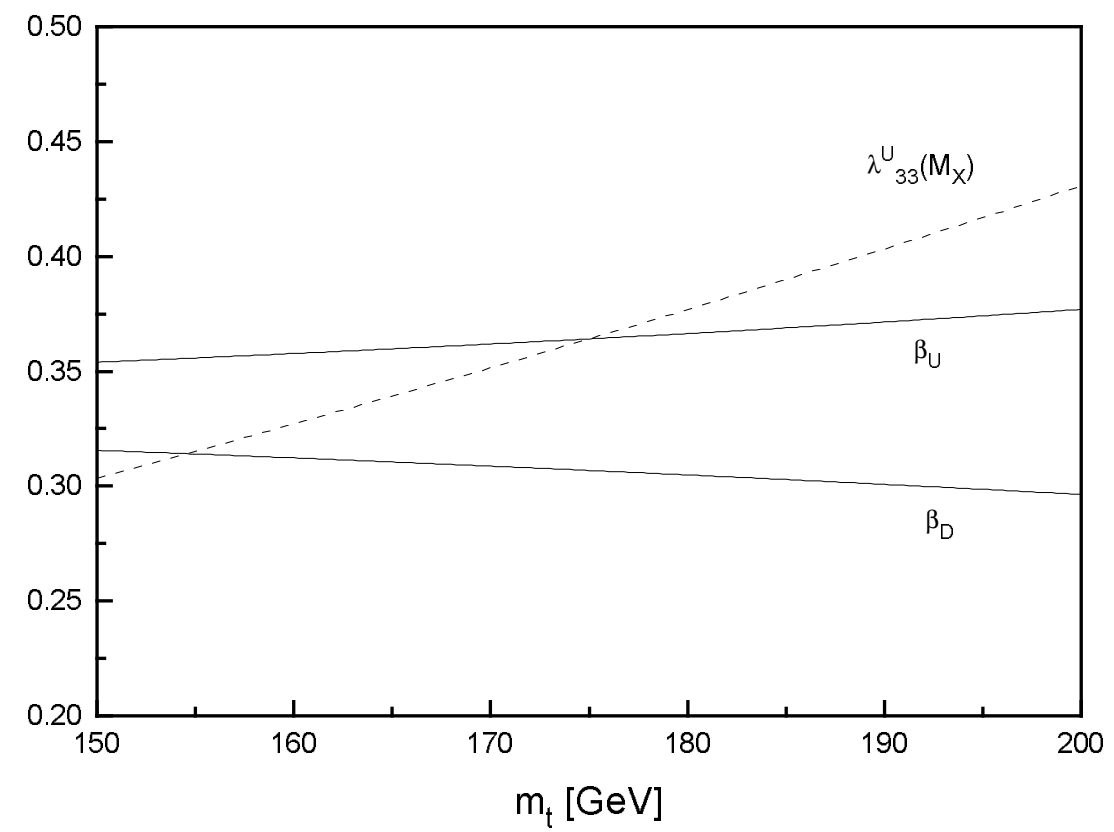

FIG. 1. Numerical results for the $\beta_{U, D}$ factors (solid lines) and $\lambda_{33}^{U}\left(M_{X}\right)$ (dashed) as functions of the top quark mass.

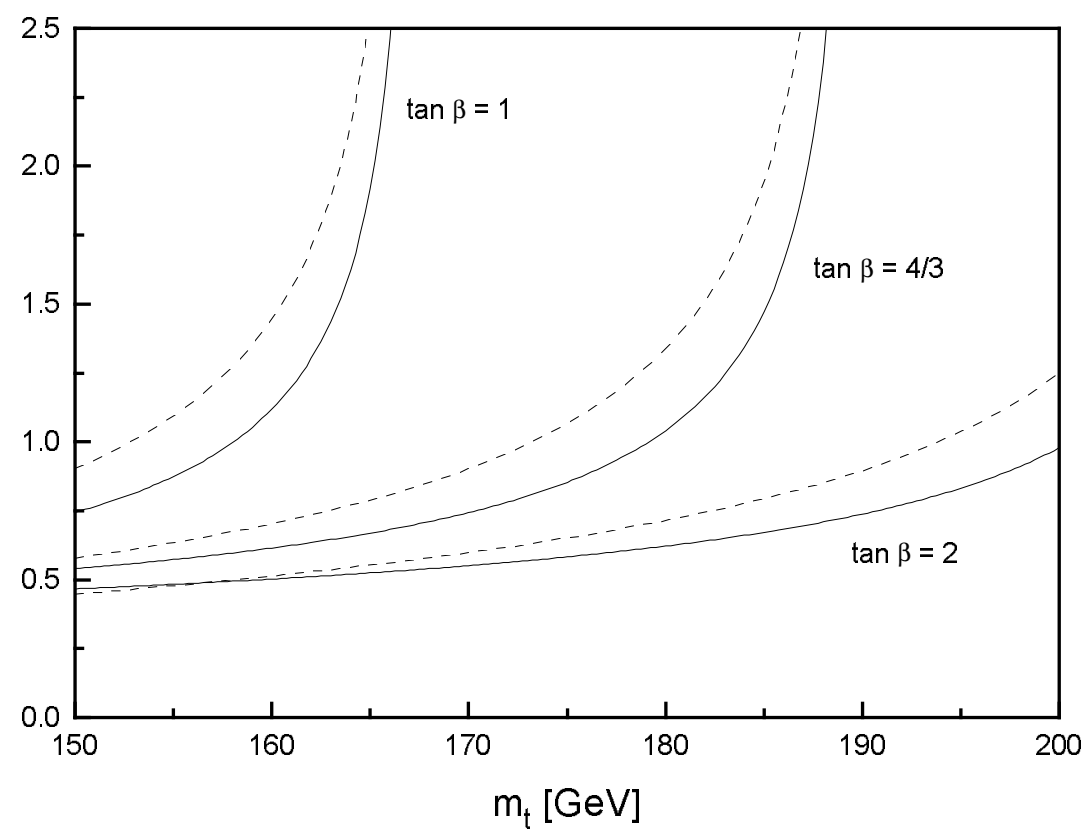

FIG. 2. Numerical results for the $\beta_{U}^{(2 h)}$ factor (solid line) and $\lambda_{33}^{U}\left(M_{X}\right)$ (dashed) in the THDM for different values of $\tan \beta$ and the top quark mass. 